\title{
Investigations on finite ideal quantum gases
}

\author{
H.-J. Schmidt ${ }^{1}$ and J. Schnack ${ }^{2}$ \\ Universität Osnabrück, Fachbereich Physik \\ Barbarastr. 7, D-49069 Osnabrück
}

\begin{abstract}
Recursion formulae of the $N$-particle partition function, the occupation numbers and its fluctuations are given using the single-particle partition function. Exact results are presented for fermions and bosons in a common one-dimensional harmonic oscillator potential, for the three-dimensional harmonic oscillator approximations are tested. Applications to excited nuclei and Bose-Einstein condensation are discussed.
\end{abstract}

PACS: 05.30.-d; 05.30.Ch; 05.30.Fk; 05.30.Jp; 03.75.Fi

Keywords: Quantum statistics; Canonical ensemble; Finite Fermi and Bose systems; Bose-Einstein condensation

\section{Introduction and summary}

In thermodynamics ideal quantum gases are usually treated in the grand canonical ensemble since all applications which were important in the past, like the electron gas, phonons or photons, deal with huge particle numbers, where the thermodynamic limit is applicable. Only the experimental attempts of the last years to investigate finite Fermi and Bose systems and to describe them in terms of thermodynamics called for theoretical effort. Interesting finite Fermi systems are for instance nuclei, which behave like a liquid drop and therefore can undergo a first order phase transition [1]. On the low excitation site of the caloric curve the nuclear systems might be well described as an ideal Fermi gas in a common harmonic oscillator potential.

1 Email: hschmidt@physik.uni-osnabrueck.de, WWW: http://www.physik.uni-osnabrueck.de/makrosysteme/hjschmidt.htm

2 Email: juergen.schnack@physik.uni-osnabrueck.de, WWW: http://obelix.physik.uni-osnabrueck.de/ schnack 
Small Bose systems became available through the development of traps. Here the focus is on the Bose-Einstein condensation which for instance could be found investigating alkali atoms in magnetic traps [2-4]. Again the system can be well described as an ideal quantum gas contained in an external harmonic oscillator potential.

Theses finite quantum systems are characterized by a constant particle number and may be represented by the canonical or the micro-canonical ensemble depending on whether heat exchange with the environment is possible or not.

In the following we present three formulae for the partition function of the canonical ensemble, one being already known [5]. All of them need the singleparticle partition function as input. In a one-dimensional harmonic oscillator the result can be further simplified for fermions and bosons, leading to the interesting result that both have the same specific heat.

In practice the exact formulae for the partition functions can be evaluated only for small particle numbers, say $N \lesssim 20$. Therefore, approximations are derived in the third section, which may be employed for larger $N$. They are tested against the exact result for small $N$. In the last section we also discuss the examples of small excited nuclei and Bose-Einstein condensation.

\section{Recursion formulae and generating functions}

Throughout this article we assume that the Hamilton operator is a single particle operator, typically the kinetic energy and a common potential or a mean field

$$
\underset{\sim}{H}=\sum_{n=1}^{N} \underset{\sim}{h}(n)
$$

The partition function $Z_{N}$ for the canonical ensemble of $N$ particles can be recursively built starting with the single-particle partition function [5]

$$
Z_{N}(\beta)=\frac{1}{N} \sum_{n=1}^{N}( \pm 1)^{n+1} Z_{1}(n \beta) Z_{N-n}(\beta), \quad Z_{0}(\beta)=1, \beta=\frac{1}{k_{B} T}
$$

where the upper sign in the sum stands for bosons, the lower sign for fermions.

A second method uses a generating function to obtain $Z_{N}$. Consider the grand canonical partition function $Q(\beta, z)$ 


$$
Q(\beta, z)=\sum_{N=0}^{\infty} z^{N} Z_{N}(\beta), \quad z=e^{\beta \mu}
$$

where $z$ is the fugacity and $\mu$ the chemical potential. Multiplying $Q(\beta, z)$ with a second function $Y(\beta, z)$

$$
Y(\beta, z)=1+\sum_{n=1}^{\infty}( \pm 1)^{n} z^{n} Z_{1}(n \beta)
$$

and grouping the result with respect to powers of $z$ yields a differential equation

$$
\frac{(1-Y(\beta, z)) \mathrm{d} z}{z}=\frac{\mathrm{d} Q(\beta, z)}{Q(\beta, z)}
$$

which can be integrated obtaining

$$
\ln \{Q(\beta, z)\}=\sum_{n=1}^{\infty}( \pm 1)^{n+1} \frac{z^{n}}{n} Z_{1}(n \beta)=: W(\beta, z)
$$

or

$$
Q(\beta, z)=\exp \{W(\beta, z)\}
$$

Expanding this function, which expresses $Q(\beta, z)$ in terms of $Z_{1}$, into powers of $z$ gives the $Z_{N}(\beta)$ as coefficients. This derivation is equivalent to first differentiating $Q(\beta, z)$ eq. (3) with respect to $z$, inserting (2) and then regrouping the sum $[6]$.

There is even a third method which represents $Z_{N}(\beta)$ explicitly in terms of $Z_{1}$

$$
Z_{N}(\beta)=\frac{1}{N !} \sum_{\nu=0}^{2^{N-1}-1}( \pm 1)^{N-k[\nu]} N[\nu] \prod_{n \in K[\nu]} Z_{1}(n \beta)
$$

We explain this method with the help of $Z_{3}(\beta)$ and table 1 . The method starts with the dual representation of $\nu$ running from zero to $2^{N-1}-1$. This string $[\nu]$ is filled with zeros at the end to get as many digits as particles, in our example three. Then this string is divided into $k[\nu]$ substrings each substring closing with exactly one zero, in our example for $\nu=1$ the string 100 is grouped into 10 and $0 . K[\nu]$ is the $k[\nu]$-tuple which consists of the lengths of those substrings. The last factor involved is $N[\nu]$ which is the product of all integers from 1 to $N$ taken to the power given by the respective digit in the string $[\nu]$. Adding up the results of our example leads to 


\begin{tabular}{c|c|c|c|c||c}
$\nu$ & {$[\nu]$} & $K[\nu]$ & $k[\nu]$ & $N[\nu]$ & result \\
\hline \hline 0 & {$[000]$} & $(1,1,1)$ & 3 & $1^{0} 2^{0} 3^{0}=1$ & $( \pm 1)^{0} 1 Z_{1}^{3}(\beta)$ \\
1 & {$[100]$} & $(2,1)$ & 2 & $1^{1} 2^{0} 3^{0}=1$ & $( \pm 1)^{1} 1 Z_{1}(2 \beta) Z_{1}(\beta)$ \\
2 & {$[010]$} & $(1,2)$ & 2 & $1^{0} 2^{1} 3^{0}=2$ & $( \pm 1)^{1} 2 Z_{1}(\beta) Z_{1}(2 \beta)$ \\
3 & {$[110]$} & $(3)$ & 1 & $1^{1} 2^{1} 3^{0}=2$ & $( \pm 1)^{2} 2 Z_{1}(3 \beta)$ \\
\hline
\end{tabular}

Table 1

Example of the explicit method eq. (8) for $N=3$.

$$
Z_{3}(\beta)=\frac{1}{3 !}\left(Z_{1}^{3}(\beta) \pm 3 Z_{1}(\beta) Z_{1}(2 \beta)+2 Z_{1}(3 \beta)\right)
$$

The proof of (8) is accomplished by regrouping the contributions $\nu$ with respect to $[\nu]$, where the first group contains all terms where $[\nu]$ ends with 00 , the second where $[\nu]$ ends with 010, the third where $[\nu]$ ends with 0110 and so on. These groups can be identified with the summands of the recursion formula (2). The coefficients of the product $\prod Z_{1}(n \beta)$ in (8) satisfy a number of combinatorial relations which will be investigated elsewhere. Here we only note, that the sum of all coefficients amounts to 0 in the fermionic case and to 1 in the bosonic case.

Following the very same idea that led to (2) one can derive recursion formulae for the occupation numbers of single-particle energy eigenstates $|n\rangle$ with energy $\varepsilon_{n}[7]$

$$
N_{n}:=\left\langle\left\langle\underset{\sim}{a_{n}^{+} a_{n}}\right\rangle\right\rangle=\frac{1}{Z_{N}(\beta)} \sum_{m=1}^{N}( \pm 1)^{m+1} \exp \left\{-m \beta \varepsilon_{n}\right\} Z_{N-m}(\beta)
$$

Any single-particle density $\rho_{N}(\beta)$ (e.g. the spatial density) is given by

$$
\rho_{N}(\beta)=\frac{1}{Z_{N}(\beta)} \sum_{n=1}^{N}( \pm 1)^{n+1} \rho_{1}(n \beta) Z_{1}(n \beta) Z_{N-n}(\beta)
$$

which follows from equation (10) using the density $\rho_{1}$ in one-particle space. For the fermion case the fluctuations are well known to be

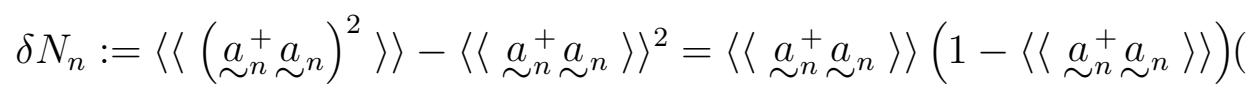

for bosons the fluctuations are given with the help of 


$$
\left\langle\left\langle\left({\underset{\sim}{\sim}}_{n}^{+} \underset{\sim}{a_{n}}\right)^{2}\right\rangle\right\rangle=\frac{1}{Z_{N}(\beta)} \sum_{m=2}^{N}(m-1) \exp \left\{-m \beta \varepsilon_{n}\right\} Z_{N-m}(\beta) .
$$

\subsection{Exact solution for the one-dimensional harmonic oscillator}

In the case of a one-dimensional harmonic oscillator potential the $N$-particle

partition functions can be given explicitly, they are (see also [8] for bosons)

$$
Z_{N}(\beta)=\exp \left(-\beta E_{0}(N)\right) \prod_{n=1}^{N} \frac{1}{1-\exp (-n \beta \hbar \omega)},
$$

which differ for bosons and fermions only in $E_{0}$

$$
\begin{array}{ll}
E_{0}(N)=N \frac{\hbar \omega}{2} & \text { for bosons } \\
E_{0}(N)=N^{2} \frac{\hbar \omega}{2} & \text { for fermions } .
\end{array}
$$

The mean energy turns out as

$$
E_{N}(T)=E_{0}(N)+\sum_{n=1}^{N} n \frac{\hbar \omega}{2}\left[\operatorname{coth}\left(n \frac{\beta \hbar \omega}{2}\right)-1\right]
$$

It is interesting to realize that ideal Fermi and Bose gases contained in a one-dimensional harmonic oscillator have the same specific heat

$$
c=\frac{1}{N}\left(\frac{\partial E(T)}{\partial T}\right)_{\omega}=\frac{k_{B}}{N T^{2}} \sum_{n=1}^{N}\left(n \frac{\hbar \omega}{2}\right)^{2} \frac{1}{\sinh ^{2}\left(n \frac{\beta \hbar \omega}{2}\right)},
$$

which is due to the equidistant spacing of energy levels and is, for instance, not valid in the case of a one-dimensional box.

\subsection{Exact solution for the one-dimensional box}

Since the formulae (2), (6) and (8) for the $N$-particle partition function rely on the knowledge of the single-particle partition function it is always valuable to have $Z_{1}$ explicitly. Considering a particle with mass $m$ in a one-dimensional box of length $L$ the energy eigenvalues are 


$$
E_{n}=\frac{\hbar^{2}}{2 m}\left(\frac{\pi n}{L}\right)^{2} \quad \text { and } \quad x:=\beta \frac{\hbar^{2}}{2 m}\left(\frac{\pi}{L}\right)^{2}
$$

will denote the dimensionless inverse temperature. Then the partition function for the one-dimensional box can be calculated as

$$
Z_{1}(\beta)=\sum_{n=1}^{\infty} \mathrm{e}^{-x n^{2}}=\frac{1}{2}\left(\vartheta_{3}\left(0, \mathrm{e}^{-x}\right)-1\right)=\sqrt{\frac{K(m)}{2 \pi}}-\frac{1}{2} .
$$

In three dimensions it is just the third power. $\vartheta_{3}$ is the third elliptic $\vartheta$-function and $K$ the complete elliptic integral [9]

$$
\begin{aligned}
\vartheta_{3}(u, q) & =1+2 \sum_{n=0}^{\infty} q^{n^{2}} \cos (2 n u), \\
K(m) & =\int_{0}^{\pi / 2} \mathrm{~d} \theta\left(1-m \sin ^{2}(\theta)\right)^{-\frac{1}{2}} .
\end{aligned}
$$

The variable $m$ is related to the dimensionless inverse temperature $x$ by

$$
m=\left(\frac{\vartheta_{2}\left(0, \mathrm{e}^{-x}\right)}{\vartheta_{3}\left(0, \mathrm{e}^{-x}\right)}\right)^{4}, \quad \vartheta_{2}(u, q)=2 q^{\frac{1}{4}} \sum_{n=0}^{\infty} q^{n(n+1)} \cos (2(n+1) u) .
$$

In order to calculate the mean energy the first derivative of $Z_{1}$ is needed which reads

$$
\frac{d Z_{1}}{d x}=-\frac{\pi^{-\frac{5}{2}}}{\sqrt{2}} K^{\frac{3}{2}}(m)[E(m)-(1-m) K(m)]
$$

with $E$ the complete elliptic integral

$$
E(m)=\int_{0}^{\pi / 2} \mathrm{~d} \theta\left(1-m \sin ^{2}(\theta)\right)^{\frac{1}{2}}
$$

\section{Approximations}

\subsection{Fermions}

The approximation rests on the fact that a three dimensional harmonic oscillator can be viewed as three independent one dimensional oscillators, one for each spatial coordinate. Then the idea is to apply the Pauli principle for 
excited states only to one of the oscillators, i.e. directions in space. The approximation of the partition function becomes [10]

$$
\begin{aligned}
& \tilde{Z}_{N}(\beta)=\exp \left(-\beta E_{0}(N)\right)\left(\frac{1}{1-\exp (-\beta \hbar \omega)}\right)^{2 N} \\
& \times \prod_{n=1}^{N} \frac{1}{1-\exp (-n \beta \hbar \omega)}
\end{aligned}
$$

with $E_{0}(N)$ being the correct ground state energy. The approximate mean energy

$$
\begin{aligned}
\tilde{E}_{N}(T)=E_{0}(N) & +2 N \frac{\hbar \omega}{2}\left[\operatorname{coth}\left(\frac{\beta \hbar \omega}{2}\right)-1\right] \\
& +\sum_{n=1}^{N} n \frac{\hbar \omega}{2}\left[\operatorname{coth}\left(n \frac{\beta \hbar \omega}{2}\right)-1\right]
\end{aligned}
$$

has besides the ground state energy three parts, two for the two directions where the Pauli principle is not applied and one where it is applied. Therefore the specific heat is a sum of two contributions for distinguishable particles in a common one-dimensional oscillator field and one for fermions in a common one-dimensional oscillator field. Although the partition function is not complete in this approximation the resulting mean energy turns out to deviate from the correct result by less then $1 \%$, see example fig. 1 . Only for $N=2$ and $N=3$ the deviation is larger. The high temperature limit is correct.
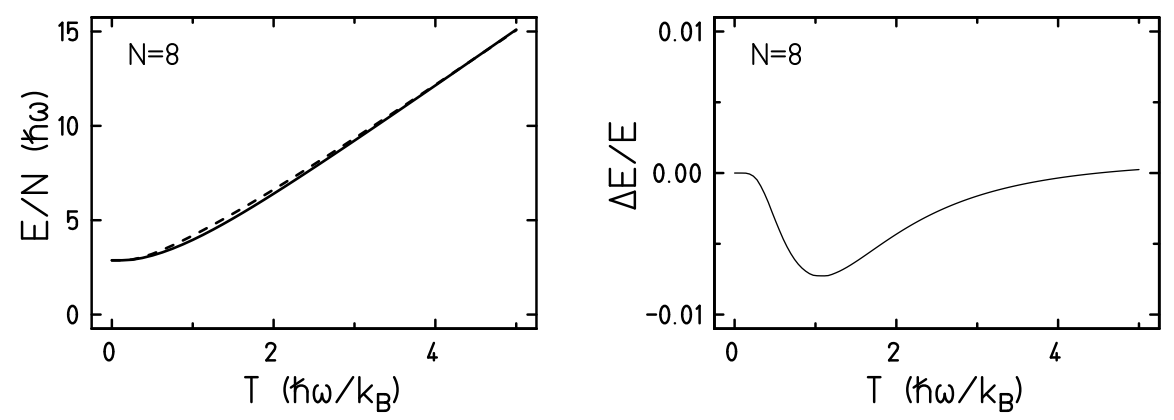

Fig. 1. L.h.s: mean energy of fermions in a three-dimensional oscillator, the solid line displays the exact result, the dashed line the approximation (26). R.h.s.: relative deviation between approximation and exact result.

\subsection{Bosons}

Unfortunately an approximation like (25) cannot be derived for bosons, here we try to derive an appropriate power series. For the ideal Bose gas in a 
three-dimensional oscillator potential we have

$$
Z_{1}=\frac{x^{3}}{\left(1-x^{2}\right)^{3}}, \quad \text { with } x:=\mathrm{e}^{-\beta \hbar \omega / 2} .
$$

Obviously, $Z_{N}$ will be a rational function of $x$. A closer inspection shows that it has the structure

$$
Z_{N}(x)=\frac{x^{3 N}}{\prod_{n=1}^{N}\left(1-x^{2 n}\right)^{3}} P_{N}(x), \quad \text { where } P_{N}(x)=\sum_{m=0}^{L_{N}} p_{m} x^{2 m}
$$

is an even polynomial with non-negative coefficients where for $N \geq 4$ the degree is $2 L_{N}=\left(3 N^{2}-7 N+10\right)$. The first $(N+1)$ coefficients $p_{0}, p_{1}, \ldots, p_{N}$ are independent of $N$, for example,

$$
P_{N}(x)=1+3 x^{4}+\cdots \quad \text { for } N>1 .
$$

Moreover, the following identities hold (the primes denote derivatives):

$$
\begin{aligned}
& P_{N}(1)=(N !)^{2} \\
& P_{N}^{\prime}(1)=\frac{3}{2} N(N-1)(N !)^{2} \\
& P_{N}^{\prime \prime}(1)=\frac{1}{12}\left(27 N^{2}-23 N-8\right) N(N-1)(N !)^{2} \\
& P_{N}^{\prime \prime \prime}(1)=\frac{1}{8}\left(27 N^{4}-42 N^{3}-9 N^{2}+16 N-20\right) N(N-1)(N !)^{2} .
\end{aligned}
$$

These identities may be proved by deriving an analogous recursion relation for the $P_{N}$ from eq. (2).

In order to approximate $Z_{N}$ we consider the function

$$
f_{N}(x)=\ln \left[\ln \left(P_{N}(x)\right)\right]
$$

which is analytic in a neighborhood of the real positive axis and has a logarithmic singularity at $x=0$ due to $(29)$. Since $f_{N}(x)-4 \ln (x)$ behaves rather smoothly in the physically relevant interval $x \in(0,1]$ it appears sensible to use a Taylor series approximation

$$
\begin{aligned}
f_{N}(x) & =: 4 \ln (x)+g_{N}(x) \\
& \approx 4 \ln (x)+\sum_{\nu=0}^{K} \frac{g_{\nu}}{\nu !}(x-1)^{\nu} .
\end{aligned}
$$


The values of the $\nu$-th derivatives $f_{N}^{(\nu)}(1), \nu=0, \ldots, K$, and hence of $g_{\nu}$, can be expressed in a straight forward way as functions of $P_{N}^{(\nu)}(1)$ and, using identities of the form (30), finally as known functions of $N$, albeit of increasing complexity. We choose $K=3$ and $K=4$ and obtain approximations $\tilde{Z}_{N}, \tilde{E}_{N}$ and $\tilde{c}_{N}$, where the latter is compared to exact results in figure 2 .
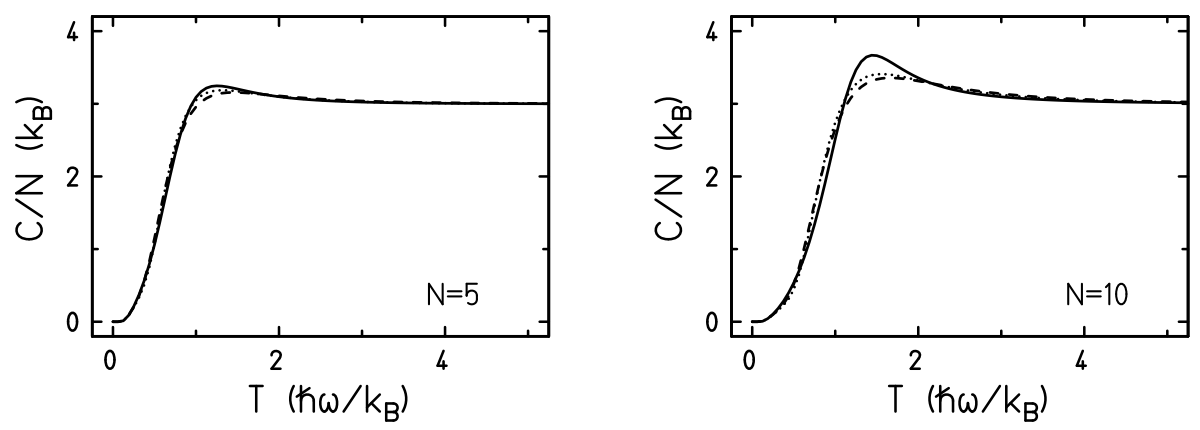

Fig. 2. Specific heat of bosons in a three-dimensional oscillator, the solid line displays the exact result, the dashed line the approximation (32) with $K=3$, the dotted with $K=4$.

One sees that the approximation already for small $K$ reflects the exact behaviour qualitatively correct. In the high and low temperature regime it works also quantitatively well, but the maximum is described only roughly both in position and heights. For larger particle numbers more and more terms of the Taylor expansion (32) would have to be taken into account.

\section{Applications}

\subsection{Fermions}

Approximation (26) enables us to evaluate the low temperature behavior of the excitation energy of nuclei consisting of for instance $A=100$ nucleons and compare it to the approximation of the free Fermi gas commonly employed in nuclear physics. Figure 3 shows on the l.h.s. as a solid line the result obtained for the common harmonic oscillator with a frequency of

$$
\hbar \omega \approx 41 \mathrm{MeV} A^{-\frac{1}{3}}
$$

which is obtained if one demands a correct root mean square radius [11]. The dashed line displays the respective dependence for the free Fermi gas with an inverse level density parameter of $10 \mathrm{MeV}$. One clearly sees that for very small excitations the temperature rises much faster with excitation energy if calculated in the oscillator shell model. Then the curve bends earlier towards 

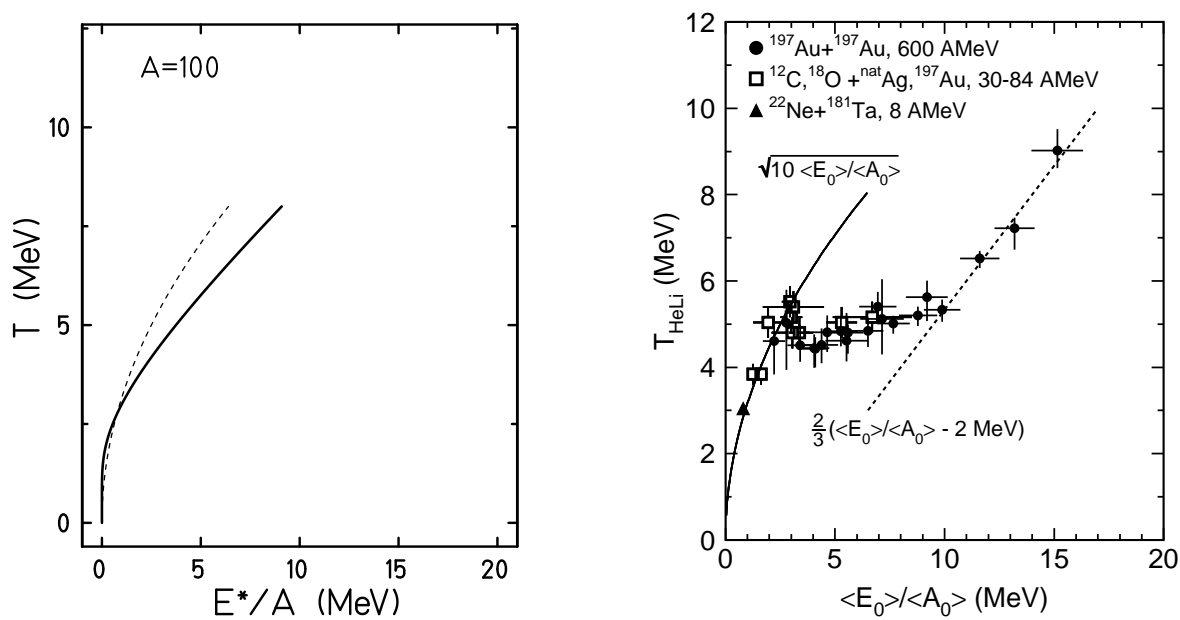

Fig. 3. L.h.s.: relation between excitation energy and temperature for the harmonic oscillator (solid line) and the free Fermi gas (dashed line). R.h.s.: experimentally determined relation, figure taken from [1].

the high temperature limit $c=3 k_{B}$, meaning that the temperature now grows less with excitation energy. In practice this observation is still beyond experimental verification as can be anticipated from the right hand side, where an attempt to determine the complete caloric curve of the nuclear liquid-gas phase-transition is displayed [1].

\subsection{Bosons}

The observation of Bose-Einstein condensation of very cold gases contained in magnetic traps has stimulated much theoretical work in this field during the last years (see for instance [12-15] and references therein). Since the investigated gases are very dilute and the trap can be modeled by a harmonic oscillator potential these systems can be viewed as ideal Bose gases in common harmonic oscillator fields.

Before going into details let us first remind some known results [16]. Rigorously speaking a phase transition towards the Bose-Einstein condensate does not happen in an external potential since the chemical potential is always nonzero for finite temperatures. Only for a vanishing potential (infinite volume limit) it is zero for all temperatures below $T_{c}$. It is also true that the phase transition does only occur for dimensions $d>2$.

Dealing with finite systems one has to relax the rigorous point of view and look for criteria that one would like to identify a "smooth Bose-Einstein condensation". A first natural criterion is the maximum in the specific heat $[17,14]$, which is a kink in the free three-dimensional case because the mean energy jumps at $T_{c}$. Looking at figures 4 and 5 (upper right corner) one sees that 

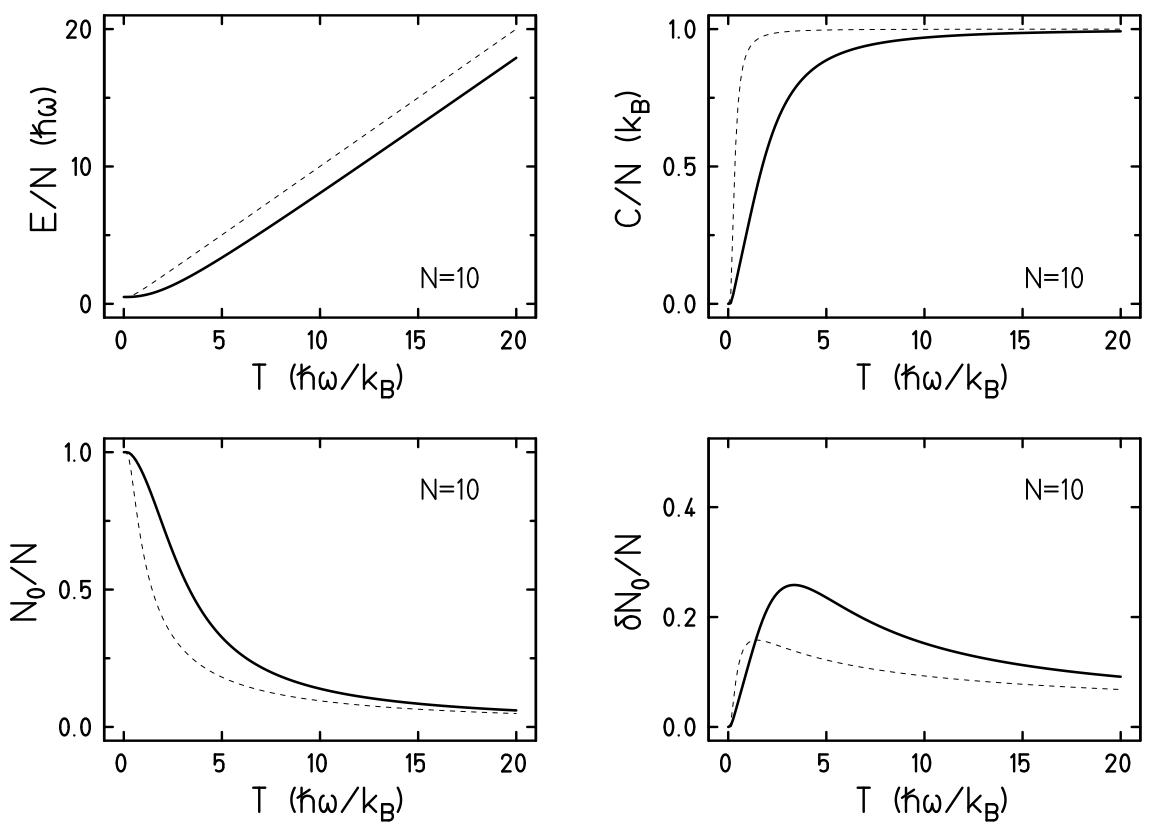

Fig. 4. One-dimensional harmonic oscillator: mean energy, specific heat, ground state occupation number and its fluctuation for bosons (solid line) and distinguishable particles (dashed line).
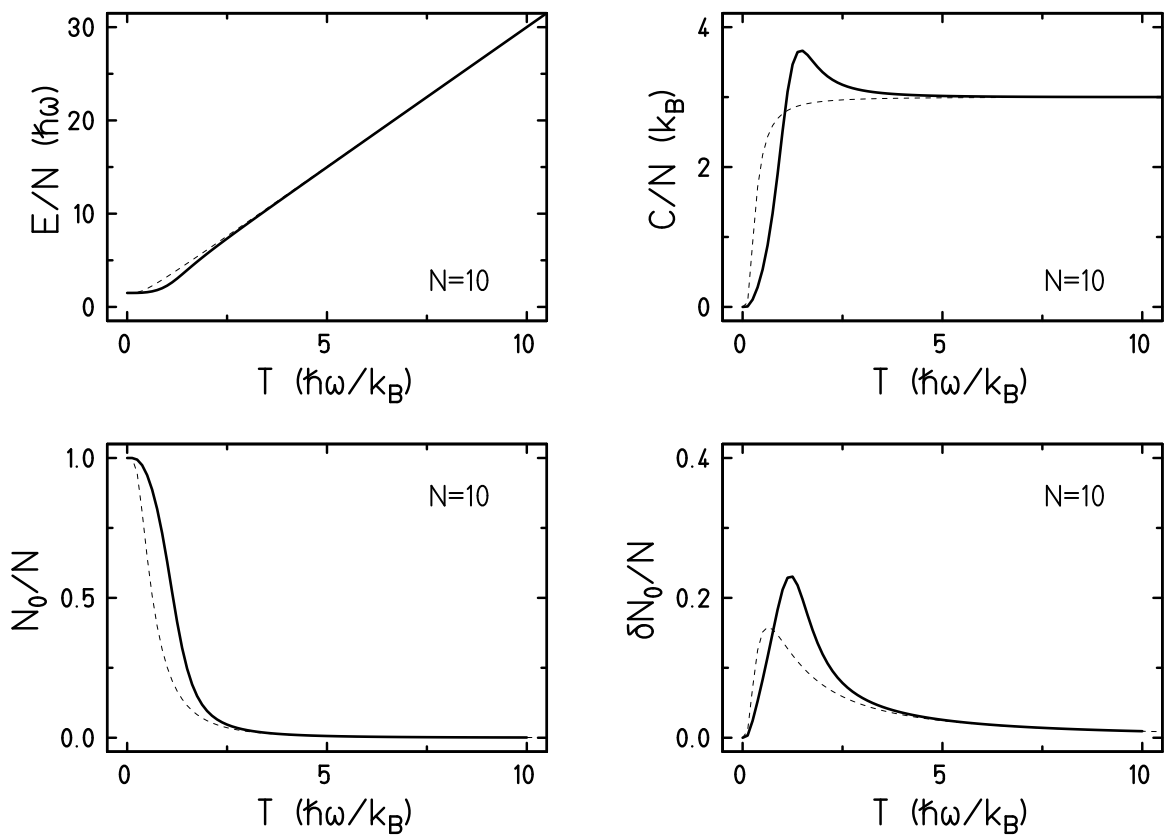

Fig. 5. Three-dimensional harmonic oscillator: mean energy, specific heat, ground state occupation number and its fluctuation for bosons (solid line) and distinguishable particles (dashed line).

the specific heat exhibits a clear maximum in the three-dimensional oscillator whereas it does not in the one-dimensional oscillator. Using the specific heat 
as a criterion for Bose-Einstein condensation the property that it does only occur for dimensions larger than two is maintained.

A second possible criterion would be the ground state occupation number $N_{0}$ and its fluctuation $\delta N_{0}$. One could argue that the ground state should be macroscopically occupied below a certain temperature $T_{c}[12]$, but as figures 4 and 5 (lower left) show, that happens also with distinguishable particles (dashed line). The same is true if one would take the existence of a maximum in the fluctuations of the ground state occupation number as a criterion. Also here the mere existence happens for distinguishable particles too, see figures 4 and 5 (lower right). One also realizes that in this respect there is no difference between the one- or three-dimensional case.
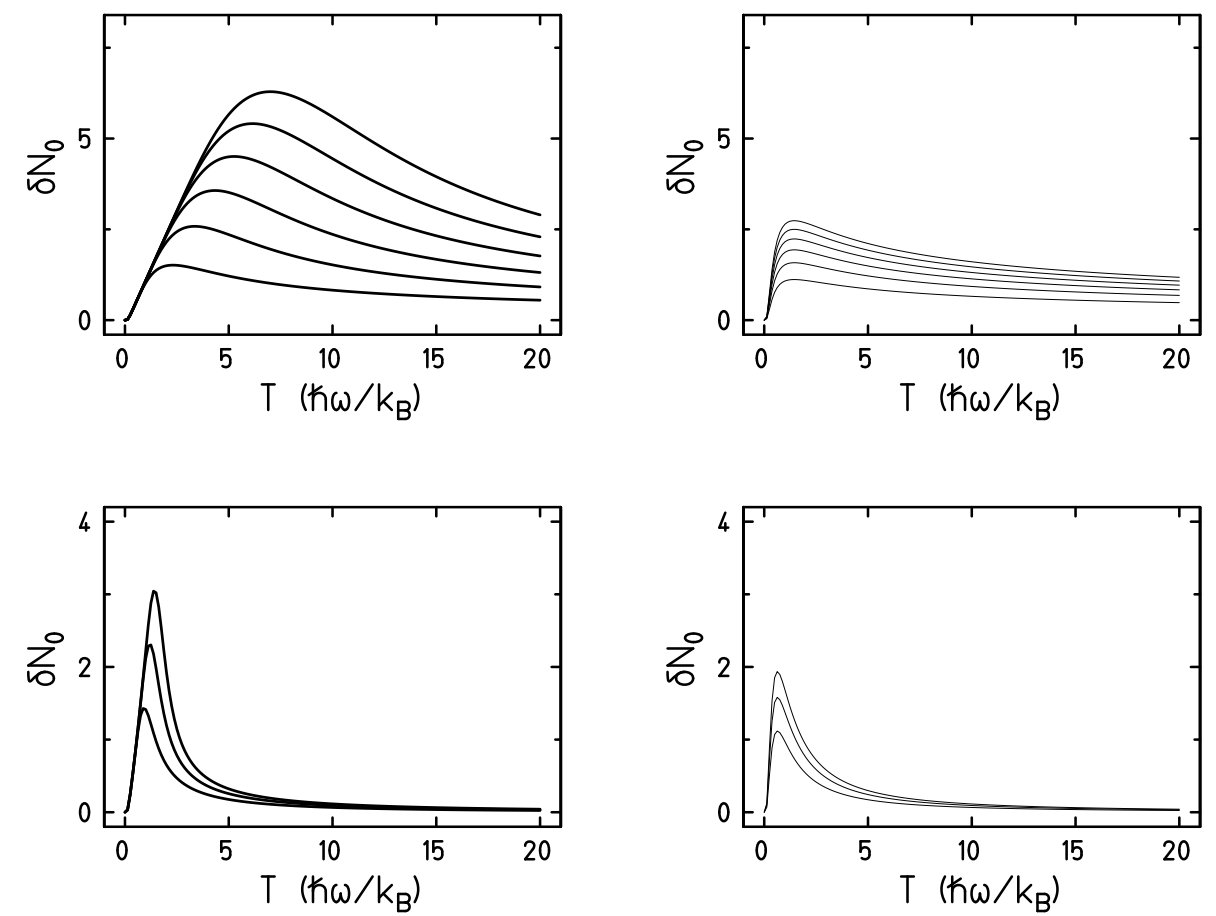

Fig. 6. Fluctuations of the ground state occupation number in a one-dimensional (upper) and a three-dimensional harmonic oscillator (lower); lines from bottom to top starting with $N=5$ in steps of 5 . L.h.s. bosons, r.h.s. distinguishable particles.

The possible key point in order to distinguish between bosons and distinguishable particles is the dependence of the magnitude of the fluctuations on the particle number. For bosons in a one-dimensional harmonic oscillator the fluctuations $\delta N_{0}$ scale with $N$ (see also [13]), whereas for distinguishable particles they scale with $\sqrt{N}$. This can also be seen in fig. 6 (upper figures).

In an experimental situation one of course knows whether one is dealing with bosons or not. The experimental interest then is to bring as many bosons as 
possible into the ground state. Comparing the one-dimensional (fig. 6 upper figures) and three-dimensional case (fig. 6 lower figures) one realizes that for the same particle number this happens at higher temperatures in one dimension.

\section{Acknowledgments}

J.S. would like to thank Jörn Knoll and Rolf Fauser for helpful advise. We thank Klaus Bärwinkel for carefully reading the manuscript.

\section{References}

[1] J. Pochodzalla et al., Phys. Rev. Lett. 75 (1995) 1040

[2] M.H. Anderson, J.R. Ensher, M.R. Matthews, C.E. Wieman, E.A. Cornell, Science 269 (1995) 198

[3] K.B. Davis, M.-O. Mewes, M.R. Andrews, N.J. van Druten, D.S. Durfee, D.M. Kurn, W. Ketterle, Phys. Rev. Lett. 75 (1995) 3969

[4] C.C. Bradley, C.A. Sackett, R.G. Hulet, Phys. Rev. Lett. 78 (1997) 985

[5] P. Borrmann, G. Franke, J. Chem. Phys. 98 (1993) 2484

[6] K. Bärwinkel, private communication

[7] P. Borrmann, dissertation, University of Oldenburg (1995) and preprint condmat/9412117

[8] F.C. Auluck, D.S. Kothari, Proc. Cambridge Philos. Soc. 42 (1946) 272

[9] E.D. Rainville, Special functions, Chelsea Publishing Company, New York (1960)

[10] J. Schnack, dissertation, TH Darmstadt (1996)

[11] P. Ring, P. Schuck, The Nuclear Many-Body Problem, Texts and monographs in physics, Springer (1980)

[12] W. Ketterle, N.J. van Druten, Phys. Rev. A54 (1996) 656

[13] S. Grossmann, M. Holthaus, Phys. Rev. E54 (1996) 3495; Phys. Rev. Lett. 79 (1997) 3557; Optics Express 1 (1997) 262

[14] K.Kirsten, D.J. Toms, Phys. Rev. A54 (1996) 4188

[15] M. Wilkens, C. Weiss, Journal of Modern Optics 44 (1997) 1801; Optics Express 1 (1997) 272

[16] K. Huang, Statistical mechanics, John Wiley \& Sons, New York (1987)

[17] H.R. Pajkowski, R.K. Pathria, J. Phys. A10 (1977) 561 